\title{
Efficacy of a Social Self-Value Empowerment Intervention to Improve Quality of Life of HIV Infected People Receiving Antiretroviral Treatment in Nepal: A Randomized Controlled Trial
}

\author{
Dharma Nand Bhatta $^{1,2} \cdot$ Tippawan Liabsuetrakul $^{2}$
}

Published online: 9 September 2016

(c) The Author(s) 2016. This article is published with open access at Springerlink.com

\begin{abstract}
We developed a comprehensive and culturally applicable empowerment intervention social self-value package with an aim to assess its efficacy in order to improve the quality of life (QoL) of HIV infected people receiving antiretroviral treatment. Participants were randomly allocated to receive either six weekly intervention sessions or standard care. Nonlinear mixed-effects models were performed to compare changes in empowerment scores over time. Between September and November 2014, 1447 individuals were screened, of whom 132 were randomly assigned to either the intervention or control group. The mean scores of empowerment, social support and quality of life increased and stigma scores were reduced in the intervention group at 3- and 6-months. An intervention effect on social support, stigma and QoL was significantly increased by time and group with low and high empowerment. No adverse events were reported. The empowerment intervention was efficacious in improving $\mathrm{QoL}$ of HIV infected people.
\end{abstract}

Resumen Hemos desarrollado un fortalecimiento completo y cultural applicable a la intervención social del

Electronic supplementary material The online version of this article (doi:10.1007/s10461-016-1546-z) contains supplementary material, which is available to authorized users.

Dharma Nand Bhatta

dnbhatta@yahoo.com

Tippawan Liabsuetrakul

ltippawa@yahoo.com

1 Department of Public Health, Nobel College, Pokhara University, Kathmandu, Nepal

2 Faculty of Medicine, Epidemiology Unit, Prince of Songkla University, Hat Yai, Thailand paquete del valor propio con la intención de evaluar su eficacia para mejorar la calidad de vida de las personas infectadas por el VIH que están recibiendo ART. A los participantes se les adjudicó aleatoriamente la asignación de seis dosis semanales o los cuidados estandar. El resultado de los efectos se presentó para comparar los cambios en los valores del fortalecimiento a lo largo del tiempo. Entre septiembre y noviembre de 2014, 1447 individuos fueron moritonizados, de los cuales 132 fueron aleatoriamente asignados para cada intervención o grupo de control. La media del valor del fortalecimiento, apoyo social y calidad de vida incrementaron y los valores del estigma fueron reducidos en la intervención grupal entre 3 y 6 meses. Los efectos de una intervención al apoyo social, estigma y calidad de vida se incrementaron significativamente en ese periodo y el grupo con un bajo y alto fortalecimiento. No hubo efectos secundarios notificados. La intervención en el fortalecimiento fue satisfactoria en la mejora de la calidad de vida de la gente infectada por el VIH.

Keywords HIV · Stigma - Quality of life · Social support · Empowerment · ART · Intervention · Adherence

\section{Background}

Epidemiological studies have highlighted decreases in HIV incidence and increased deaths related to HIV/AIDS [1-5]. However, the prevalence of HIV has remained extensive and epidemic contained enormous heterogeneity [4]. Decreasing trends of epidemics and increased life expectancy of people infected with HIV have been reported but risky sexual behaviors have promoted the transmission of HIV among general populations, and re-infections and co- 
infections among HIV infected people [3, 6]. Disease burdens are reported to be more common among HIV infected populations compared to general populations, altering their quality of life (QoL) [7-9]. The life expectancy among people living with HIV after they have initiated suitable antiretroviral treatment (ART) or combined antiretroviral treatment is reported to be similar to the general population $[10,11]$.

Availability of the ART among HIV infected people is very low and combined with psychological distress might lead to the development of anxiety, low QoL and increased stigma [4, 12]. The effect of ART on QoL was found to be reasonable among HIV infected people [13-15]. Further, their quality of life is influenced by health status, economic factors and psychological status [16]. The mechanism of how immunological and virological response influences quality of life among HIV infected people has not been comprehensively studied and therefore the effect of HIV status on QoL is unclear. Albeit, much less has been identified about the QoL of HIV infected people in comparison with other people despite considerable progress in medical prospective [16, 17]. Moreover, the effect of treatment on HIV might be affected by several factors which would help to increase stigma, reduce QoL and social support and disempowerment of HIV infected people [18-21].

Social taboos and stigma are universal socio-cultural barriers for HIV control and prevention [22, 23]. Empowerment and social support could be helpful assets among HIV infected people to enhance their QoL, reduce stigma and improve adherence to ART in resource poor settings $[24,25]$. A practical and integrated program is needed for empowerment of HIV infected people [26, 27]. Empowerment would be best approached to reduce HIV risk associated problems with cost effective interventions [28-30]. However, HIV related empowerment based programs are sparse and it has been necessary to strengthen and implement them with usual ART and other programs with political and social transforms [26, 31-33].

Different organizations have set the goal to achieve zero discrimination, transmission and stigma but the interventions are too sparse to cover these goals [2, 34, 35]. In addition, the '90-90-90' target has been set by UNAIDS by the year 2020 [36]. Therefore, extensive socially and culturally accepted cost-effective interventions are needed which enhance QoL and social support, and eliminate stigma among HIV infected people in resource poor settings [15, 37-40]. Unfortunately, most of the interventions were established in developed countries and few from developing countries. There is thus an urgent need to develop a culturally sensitive intervention for use in developing countries. An empowerment intervention program we have developed, designed to improve QoL of HIV infected people, was developed on the foundation of the diffusion model of innovations [41], and followed different theories to connect empowerment framework that assumed to change behavior, self-esteem, social support, discrimination, stigma and QoL [6, 42-52]. The aim of this study was to assess the efficacy of a social self-value empowerment intervention package to improve QoL of HIV infected people receiving ART. Furthermore, we assessed the effect of this intervention to enhance social support and reduce stigma.

\section{Methods}

\section{Study Design, Settings and Participants}

In this open label, parallel, randomized controlled trial, HIV infected participants receiving ART from the ART center in Kathmandu, Nepal were recruited. The study was carried out between September 2014 and June 2015 in Sukraraj Tropical and Infectious Disease Hospital (STIDH), Teku, Kathmandu, which is administered by the National Center for AIDS and STD Control (NCASC) [53]. STIDH is the largest ART center in the country that has been providing multidisciplinary medical services for all HIV infected people since 2004 [54]. Details of the study participants recruitment and design are presented in Fig. 1.

To be included in the study, participants had to be HIV infected, aged 18 years or older, and have been receiving ART between 6 months and 2 years prior to the study as per the national ART guidelines of NCASC [55]. Participants with severe health problems (including psychotic disorders, visual and hearing problems), had attended similar intervention programs or any other education

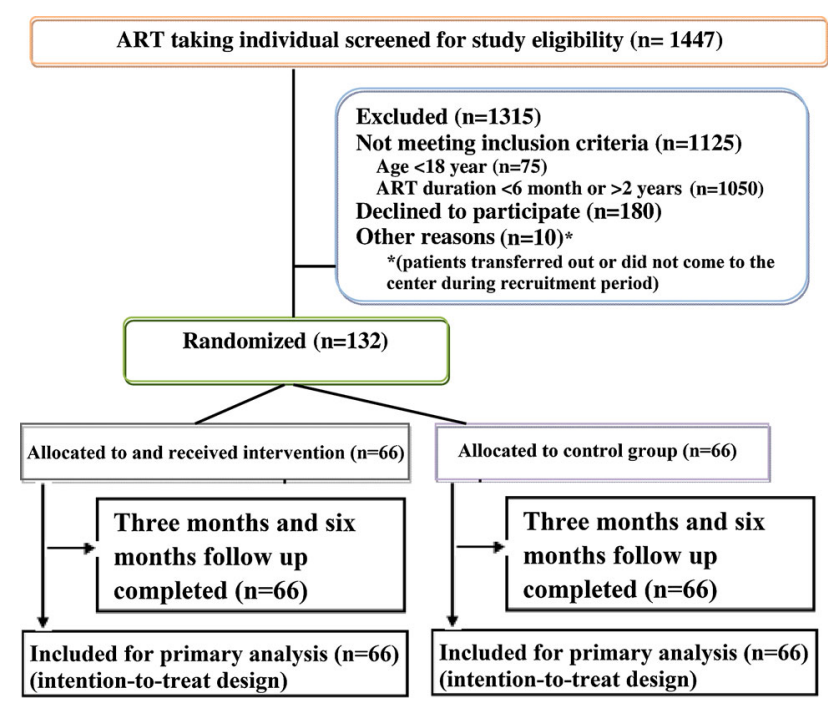

Fig. 1 Study design and participant enrolment flow diagram 
programs, were unable to attend all the study follow up visits, or were unwilling to disclose their HIV status among other participants were excluded from the study.

We calculated that 132 participants (66 in each group) would achieve $80 \%$ power to detect $20 \%$ mean difference in QoL scores between the two groups with a confidence interval of $95 \%$.

\section{Randomization and Masking}

Eligible participants were randomly allocated (1:1) to receive either the intervention sessions or standard care. Randomization was performed by a random number generator with permuted blocks of six. Allocation concealment was done by using sequentially numbered opaque sealed envelopes. The random number sequence was generated by an independent data manager. Members of the research team and participants were masked to these numbers and the randomization process. None of the participants were allowed to modify their assignments after randomization. The statistician and research staff doing the baseline and follow up assessments were masked to assignment of participants by using a unique code system. Enrollment, randomization and intervention sessions were conducted between September and November 2014. First follow up assessments were done after 3 months from baseline (January-February, 2015) and 6 months follow-up assessments were done 3 months from the first follow up (MayJune, 2015).

\section{Intervention Procedures}

Baseline information was collected after recruitment and allocation of the participants. The intervention was delivered over six sessions held weekly at the ART center lasting one and half hours. Sessions were conducted with a group of 8-10 participants. All the intervention sessions were facilitated by two national level trainers with a public health graduate degree. A facilitator delivered the intervention with participatory learning activities, buzz sessions, brain storming, lecture, and discussion techniques. Participants were encouraged and motivated to communicate and discuss with different people about prevention, treatment and disclosure of HIV issues [56, 57].

The development of the intervention contents involved review of existing literature that followed social learning and action theory and empowerment principles for HIV prevention and treatment [6, 42-52]. Culturally accepted and adopted components were developed after several consultations with experts and pre-tested among HIV infected people. Based on the findings from consultants and pre-testing, a complete manual for execution of a 6 week group intervention was developed by the research team.
The empowerment intervention mainly focused on autonomy and community activism, self-esteem/self-efficacy, self-care, optimism and control over the future, family and social relationships, power-powerlessness, management of stress and righteous anger, stigma and discrimination issues, legal provisions, and human and health rights. Details of the empowerment intervention contents are available in Additional Table 1.

Briefly, all intervention sessions were started with group and ground rules, formal opening and closing custom, sharing and discussion. The first session started with rapport building, emotions, sharing uncomfortable situations and management of negative feelings and anger. The second session focused on barriers and strategies of HIV disclosure, self-esteem/self-respect/self-worth, stigma and defeat with stigma. The third session involved discussions about healthy body with healthy mind, healthy sexual relations, means to be HIV infected or non-infected and to be a man or woman, optimism and control over the future, sexuality, adherence of ART and other treatment and prevention options. The fourth session involved educated strategies for planning healthy relations with family members, the community and society, ways of effective communication and maintaining healthy relations, autonomy and community activism, and roles and responsibilities in the society. The fifth session involved education about the effects of alcohol consumption, drug use, smoking, developing skill to prevent co-infection, re-infection and risky sexual behavior, diet and exercise. In the sixth session, participants were educated about legal empowerment, human rights, legal protection, powerlessness, discrimination, stress, freedom of voice against discrimination, health rights and future goals [56].

Fidelity of the intervention was maintained with continuous monitoring of the allocated time for topic, methods and contents of the sessions by a health officer and supervised by the research team leader. Participants were assured to receive equal chances on discussion with privacy. A checklist was developed to maintain fidelity of the intervention. The checklist included intervention contents (each session had different contents), time allocated for each activity, participants interaction with listening, openness, attentiveness, engagement, understanding and reinforcement and an agenda for the next session. The percent of items rated as "appropriate" by the reviewer was more than $95 \%$, however we had only one reviewer due to limited manpower in the government agency so we could not calculate the level of agreement. A debriefing session was conducted at the end of each session for the feedback from the reviewer and facilitators. Intervention sessions were not gender-separated. We measured the acceptability using a session evaluation form (SEF) [58] and satisfaction of the participants in the intervention using 
Table 1 Clinical and behavioral characteristics

\begin{tabular}{|c|c|c|c|}
\hline & Control group $(n=66)$ & Intervention group $(n=66)$ & $p$ value \\
\hline \multicolumn{4}{|l|}{ Age at HIV diagnosis } \\
\hline Median (IQR) & $33(26.5,41)$ & $33(30,40)$ & 0.56 \\
\hline$\leq 33$ years & $34(51.5)$ & $34(51.5)$ & 0.10 \\
\hline$>33$ years & $32(48.5)$ & $32(48.8)$ & \\
\hline \multicolumn{4}{|l|}{ Age at ART initiation } \\
\hline Median (IQR) & $35(28,42.8)$ & $35(30.2,40.8)$ & 0.55 \\
\hline$\leq 35$ years & $36(54.5)$ & $38(57.6)$ & 0.86 \\
\hline$>35$ years & $30(45.5)$ & $28(42.4)$ & \\
\hline Duration of ART & & & 0.60 \\
\hline$<1$ year & $38(57.6)$ & $34(51.5)$ & \\
\hline$\geq 1$ years & $28(42.4)$ & $32(48.5)$ & \\
\hline Spouse HIV status & & & 0.15 \\
\hline Negative & $16(27.6)$ & $26(41.9)$ & \\
\hline Positive & $42(72.4)$ & $36(58.1)$ & \\
\hline Mode of HIV transmission & & & 0.16 \\
\hline Others & $34(51.5)$ & $25(37.9)$ & \\
\hline Sex-worker & $32(48.5)$ & $41(62.1)$ & \\
\hline Sexual intercourse in last 3 months & & & 1.00 \\
\hline Yes & $50(75.8)$ & $50(75.8)$ & \\
\hline No & $16(24.2)$ & $16(24.2)$ & \\
\hline Extra-marital sex & & & 0.78 \\
\hline Yes & $7(14)$ & $9(18)$ & \\
\hline No & $43(86)$ & $41(82)$ & \\
\hline Clinical stage & & & 1.00 \\
\hline I and II & $34(51.5)$ & $35(53)$ & \\
\hline III and IV & $32(48.5)$ & $31(47)$ & \\
\hline Known co-morbidities & & & 0.80 \\
\hline Tuberculosis & $10(15.2)$ & $8(12.1)$ & \\
\hline Other & $56(84.8)$ & $58(87.9)$ & \\
\hline
\end{tabular}

a client satisfaction questionnaire (CSQ-8) [59] at the end of the intervention. All the items in the SEF indicated a higher level acceptability among the intervention group. The mean score of all items ranged from 3.68 to 3.82 with a standard deviation of 0.39 to 0.47 . The total score indicated that participants either agreed or strongly agreed with the sessions. The level of participant satisfaction was high. All the participants stated that the quality of the intervention was excellent. The majority of participants $(92.4 \%)$ were very satisfied with the amount of help provided to them. Almost all $(95.5 \%)$ agreed to join the program again and were willing to refer it to others (data not shown).To maintain compliance, at the end of each session the counselors motivated participants to participate in the next session, encouraged voluntary independent participation and provided gift vouchers. The overall retention rate was $96.6 \%$ in the intervention session. All the participants were compensated for each of their six sessions with an equivalent of USD 20.

\section{Standard Care}

All participants received routine standard care as per the NCASC guidelines [55]. This included pre ART counseling, routine medical and laboratory tests and monthly follow up for ART. Standard care in Nepal is provided by government organizations and ART is dispensed free of charge.

\section{Study Procedures}

Participants were asked to provide information on clinical and behavioral characteristics, QoL, stigma, social support and empowerment at baseline, and at the scheduled 3- and 6-months follow up visits. To minimize data entry errors and enhance quality control, double data entry was employed and extensively supervised by the research team leader. Anonymity and confidentiality were maintained with assigned unique codes at randomization, baseline and 
follow up. The intervention protocol and tools were pretested among ten HIV infected people before data collection. All tools were translated into Nepali and back translated into English and appeared culturally suitable to the experts.

\section{Outcomes}

Background, behavioral and clinical characteristics of the participants were collected including age at HIV diagnosis, age at ART initiation, HIV status of spouse, mode of HIV transmission, sexual intercourse in the last 3 months and extra-marital, clinical stage, co-morbidity, adherence to ART (coded as yes or no) and empowerment. The primary outcome was QoL. Secondary outcomes were stigma and social support.

QoL was measured using WHOQoL-HIV [60] which contains 29 items divided into six domains, namely physical, psychological, level of independence, social, environmental and spiritual. It has also one general item score that measures overall quality of life and general health. All the items were rated using a 5-point Likert scale where 1 indicated low or negative perceptions and 5 indicated high or positive perceptions. Higher scores indicated better quality of life. All the domain scores were obtained by adding the component means in the individual domain, and dividing by the number of components in that domain, and multiplying by 4 , so that scores ranged from 4 (worst possible QoL) to 20 (best possible $\mathrm{QoL}$ ).

Social support was measured using the social support questionnaire number (SSQN) and social support questionnaire satisfaction (SSQS) scales [61]. SSQN indicates number of supportive persons and SSQN indicates satisfaction with available social support. Both domains included six questions. The SSQN collected the number of supportive persons that denotes different types of social support. The SSQS were rated using a 6-point Likert scale ranging from very dissatisfied to very satisfied with available support. Higher SSQN scores indicated a perceived higher level of supportive persons and higher SSQS scores indicated higher level of satisfaction from available support.

Stigma was measured using a 23-item scale questionnaire [62]. Each item was rated using a 4-point agreement scale ranging from strongly disagree to strongly agree. Total stigma scores ranged from 23 to 92 . There were three subscales, namely shame/blame/social isolation (10-40 score), perceived discrimination (8-32 score) and equity (5-20 score).

Empowerment was measured using a 28 -item scale questionnaire [63] containing a 4-point agreement scale ranging from strongly disagree to strongly agree. Total scores ranged from 28 to 112 . Empowerment was then classified into low or high using the first quartile score as the cut-off value to ensure the sample sizes were adequate.

\section{Statistical Analyses}

Each study group's clinical and behavioral characteristics measured were initially compared using Chi square tests or Fisher's exact test for categorical outcomes and Wilcoxon's signed rank test and unpaired $t$-tests for continuous outcomes, as appropriate.

Nonlinear mixed-effects regression models were used to evaluate the effect of the intervention on the primary and secondary outcomes. Covariates included empowerment, adherence to ART, age, sex, time (baseline, 3-, or 6-months follow-up), and group-by-time interactions. The mixed effect model was used to adjust for the underestimation of variances in analysis for longitudinal data [64]. The effects of empowerment, social support and stigma on the QoL were also analyzed with a mixed effect model.

Nonparametric mixed-effects regression models were also used to evaluate the relative intervention effects on social support, stigma and QoL with and without stratification by empowerment. Relative intervention effects with $95 \%$ confidence intervals with and without stratification by empowerment level among both intervention and control groups at baseline, 3- and 6-months follow up were presented. Estimated improvements with $95 \%$ confidence intervals were plotted.

All statistical analyses were conducted with R software [65]. $p$ values less than 0.05 were considered to be significant.

\section{Ethical Considerations}

Extensive anonymity, confidentiality and privacy were maintained during the recruitment, intervention and data collection process. Confidentiality and safety of the intervention study data were maintained as per the standard protocol [66]. National guidelines and principles of Nepal health research council, and the declaration of Helsinki were followed to obtain written informed consent and enrollment of the participants. Participants were fully informed about time, methods, and their right to withdraw at any time and skip any question for any reason. Reimbursement for travel cost during the intervention and follow up period were provided to all the participants. No conflict of interest and no direct or indirect financial benefits were anticipated by researchers in this intervention.

Research Ethics Committee, Faculty of Medicine, Prince of Songkla University, Thailand (57-0146-18-5) and Institutional Ethical Review Committee of STIDH, Nepal (063/071/72) approved this study. The trial was registered 
Table 2 Mean values of empowerment, stigma, social support and quality of life scores among HIV infected people

\begin{tabular}{|c|c|c|c|c|c|c|}
\hline & \multicolumn{2}{|l|}{ Baseline } & \multicolumn{2}{|c|}{3 months follow up } & \multicolumn{2}{|c|}{6 months follow up } \\
\hline & Control & Intervention & Control & Intervention & Control & Intervention \\
\hline Empowerment & 46.70 & 46.38 & 48.23 & 94.68 & 46.53 & 95.92 \\
\hline \multicolumn{7}{|l|}{ Social support } \\
\hline TSSQN & 15.04 & 14.70 & 16.92 & 28.09 & 18.58 & 30.70 \\
\hline TSSQS & 15.04 & 15.42 & 17.44 & 29.00 & 19.44 & 32.67 \\
\hline Overall stigma & 76.03 & 76.50 & 72.91 & 39.41 & 73.03 & 38.26 \\
\hline Shame/blame/social isolation & 33.11 & 33.64 & 32.21 & 16.89 & 31.27 & 16.76 \\
\hline Perceived discrimination & 26.00 & 26.17 & 24.36 & 14.45 & 24.95 & 13.86 \\
\hline Equity & 16.92 & 16.70 & 16.33 & 8.06 & 16.80 & 7.64 \\
\hline Overall quality of life & 7.76 & 7.68 & 8.46 & 15.47 & 8.15 & 15.81 \\
\hline Physical & 8.06 & 7.89 & 8.59 & 15.74 & 8.23 & 15.88 \\
\hline Psychological & 7.78 & 7.76 & 8.10 & 16.04 & 8.05 & 15.99 \\
\hline Independence & 7.42 & 7.32 & 8.58 & 15.51 & 8.32 & 15.65 \\
\hline Social relations & 7.61 & 7.53 & 8.74 & 15.47 & 8.17 & 15.82 \\
\hline Environment & 7.48 & 7.48 & 8.20 & 15.14 & 7.98 & 15.68 \\
\hline Spiritual/religious/personal belief & 8.45 & 8.27 & 8.88 & 15.12 & 8.39 & 15.92 \\
\hline General overall health & 11.97 & 11.58 & 10.64 & 13.76 & 9.88 & 15.03 \\
\hline
\end{tabular}

$T S S Q N$ total social support with number, TSSQS total satisfaction with social support

through Thai Clinical Trial Registry with registration number TCTR20140814002 (www.clinicaltrials.in.th).

\section{Results}

A total of $1447 \mathrm{HIV}$ infected people receiving ART were screened from September to November 2014, of which 1135 were ineligible due to age $<18$ years $(n=75)$, duration of ART $<6$ months or $>24$ months $(n=1050)$, and others $(n=10) .180$ eligible participants refused to join the study, giving a response rate of $42.3 \%$. Finally, 132 participants were recruited and randomly assigned to the control group $(n=66)$ or intervention group $(n=66)$. The diagram of participant flow is presented in Fig. 1. All the recruited participants completed the study at baseline, 3- and 6-months follow up. The overall retention rate was $96.6 \%$. No unfavorable events were reported during the study period.

The mean age of the participants was $36.1(\mathrm{SD}=7.8)$ years. Most of the participants were female (53\%), nonindigenous $(56.1 \%)$, could read and write only $(28.8 \%)$, and were married (74.2\%). The majority $(84.8 \%)$ of participants had an average of two children and there were no significant differences at baseline between the control and intervention groups. Table 1 compares baseline behavioral and clinical characteristics between participants in the two groups. More than $50 \%$ of participants were diagnosed with HIV before 33 years of age among both control and intervention groups. Two thirds of participants $(62.1 \%)$ in the intervention group were infected through sexual contact. Three fourths $(75.8 \%)$ were sexually active within the last 3 months. No significant differences of behavioral and clinical characteristics were detected between the two groups.

The mean empowerment, social support, stigma and QoL scores are presented in Table 2. All the scores at baseline were equally static in both groups. The mean scores of empowerment and QoL increased two-fold in the intervention group at 3 months but no further increase at 6 months. Stigma scores were reduced by half at 3 months in the intervention group with no further changes at 6 months. Social support scores increased by 1.5 times higher at 3 months follow up in the intervention group compared to the control group. Overall mean QoL scores at 3 months increased by $80 \%$ in the intervention group. The relative intervention effects among total social support with number (TSSQN), total social satisfaction with support (TSSQS), stigma and QoL had a similar trend in the intervention group (Fig. 2). Minimal improvements of outcomes were observed in the control group. Figure 3 reveals the relative intervention effects of social support, stigma and QoL by level of empowerment. The effects of the intervention on social support, stigma and QoL persisted at 6 months regardless of level of empowerment (high vs. low).

Table 3 presents the outcomes from the nonlinear mixed-effects regression model. Empowerment 

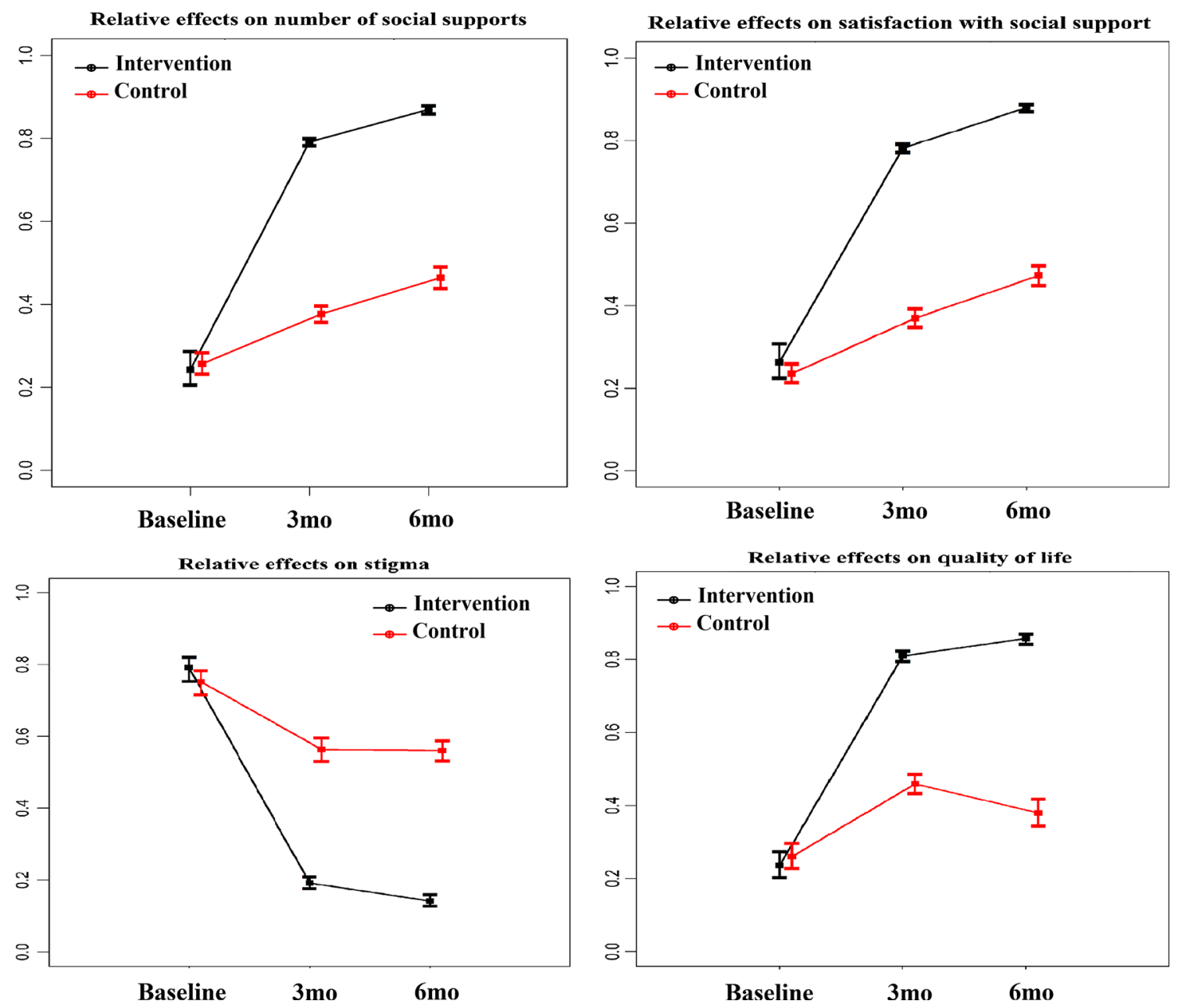

Fig. 2 Relative intervention effects on social support, stigma and quality of life using nonlinear mixed-effect model. All $p$ values for time trend between intervention and control on each outcome were

significantly reduced stigma and increased QoL $(p<0.001$ and $p<0.001)$ after adjusting for age, gender, adherence to ART, group and time. There was no significant difference in any outcome between the intervention and control group at baseline. There were significant interaction effects of intervention by time indicating that improvements in social support and QoL for the intervention group were significantly higher compared to the control group over time $(p<0.001)$, while stigma was significantly lower $(p<0.001)$. Estimated differences in improvement in social support, stigma and QoL at 3- and 6-months from baseline between intervention and control were significant (all $p$ values $<0.001)$. Increasing ART adherence was associated with a reduction of stigma $(p<0.001)$. Age and gender were not significantly associated with any outcome.

Predictors of overall QoL are presented in Table 4. Increased empowerment had a significantly higher level of QoL $(p<0.001)$. Increased stigma had a lower level of QoL but this was not statistically significant. Social support

significantly different with $p<0.001,3 m o$ three months follow up, 6mo six months follow

had no significant effect on QoL. Improvement in QoL remained statistically significant at 3- and 6-months follow up $(p<0.001)$.

\section{Discussion}

Empowerment, social support, stigma and QoL of HIV infected people at baseline were low among both groups. The improvement of social support, stigma and QoL was seen immediately after 3 months among those in the intervention group and persisted for another 3 months. The intervention positively affected social support, stigma and QoL in the equivalence regardless of level of baseline empowerment. Empowerment significantly affected social support, stigma and QoL but only empowerment was shown to be a significant predictor of QoL in addition to the intervention. 



Fig. 3 Relative intervention effects on social support, stigma and QoL stratified by empowerment using nonlinear mixed-effect model. All $p$ values for time trend between intervention and control on each

outcome were significantly different with $p<0.001$, 3mo three months follow up, 6 mo six months follow up

Clinical and behavioral characteristics were not significantly different at baseline between the two groups. Recruitment process followed strong protocol that assured the high retention rate in the intervention and lower loss to follow up. Most eligible participants declined to participate in the study; however, there was no difference in their background characteristics compared to those who participated in the study. Reasons for refusal to participate in the study were: too busy, unable to manage time for all the intervention sessions, lack of interest in the study and unavailability for follow up.

Social support was significantly improved by enhancing empowerment in the intervention group. As far as in our knowledge, empowerment interventions for all the HIV infected population were not available and comparison of the results with this trial would be difficult. A randomized controlled trial using a group support psychotherapy for HIV infected people as an intervention and measured at the same period as our study also showed an increasing social support after intervention by time [67]. Slightly improved social support in the control group at 3 months might be explained by learning process of participants from the repetitive questionnaires inducing their behavior changes (pretest sensitization effect) and possible effects of the contamination [68]. Stigmatization, discrimination, and cognitive state-the psychological condition that is characterized by a lack of obvious and logical belief and behavior-might be removed with increased social support through empowerment.

Overall stigma decreased after the intervention, more so in the intervention group. This finding was supported by the conclusion of systematic reviews that focused on any 
Table 3 Effect of empowerment intervention on social support, stigma and quality of life using nonlinear mixed-effects regression model

\begin{tabular}{|c|c|c|c|c|c|c|c|c|c|c|c|c|}
\hline \multirow[t]{2}{*}{ Parameter } & \multicolumn{3}{|l|}{ TSSQN } & \multicolumn{3}{|l|}{ TSSQS } & \multicolumn{3}{|l|}{ Stigma } & \multicolumn{3}{|c|}{ Quality of life } \\
\hline & Estimate & SE & $p$ value & Estimate & SE & $p$ value & Estimate & SE & $p$ value & Estimate & SE & $p$ value \\
\hline Empowerment & -0.010 & 0.038 & 0.795 & -0.024 & 0.040 & 0.544 & -0.127 & 0.038 & $<0.001$ & 0.057 & 0.009 & $<0.001$ \\
\hline Age & -0.001 & 0.028 & 0.997 & -0.038 & 0.029 & 0.196 & 0.005 & 0.016 & 0.744 & 0.005 & 0.005 & 0.289 \\
\hline Male versus female & 1.196 & 0.449 & 0.008 & 1.215 & 0.464 & 0.009 & 0.315 & 0.250 & 0.208 & -0.009 & 0.074 & 0.896 \\
\hline ART adherence & -0.681 & 0.393 & 0.083 & -0.424 & 0.409 & 0.300 & -1.300 & 0.402 & $<0.001$ & 0.145 & 0.105 & 0.151 \\
\hline $\begin{array}{l}\text { Intervention versus control } \\
\text { at baseline }\end{array}$ & -0.496 & 0.532 & 0.351 & 0.243 & 0.550 & 0.659 & 0.388 & 0.425 & 0.360 & -0.060 & 0.109 & 0.579 \\
\hline Time (months) & & & $<0.001$ & & & $<0.001$ & & & $<0.001$ & & & $<0.001$ \\
\hline Intervention $\times$ Time & & & $<0.001$ & & & $<0.001$ & & & $<0.001$ & & & $<0.001$ \\
\hline \multicolumn{13}{|c|}{ Difference in improvement from baseline between intervention and control group } \\
\hline 3 months follow up & 11.983 & 1.868 & $<0.001$ & 12.304 & 1.936 & $<0.001$ & -28.028 & 1.890 & $<0.001$ & 4.430 & 0.474 & $<0.001$ \\
\hline 6 months follow up & 12.967 & 1.977 & $<0.001$ & 14.041 & 2.049 & $<0.001$ & -28.927 & 1.998 & $<0.001$ & 4.904 & 0.501 & $<0.001$ \\
\hline
\end{tabular}

$S E$ standard error, TSSQN total social support with number, TSSQS total satisfaction with social support

Table 4 Prediction of QoL by intervention group after adjusting for level of empowerment, social support and stigma using nonlinear mixed-effects regression model

\begin{tabular}{lcrr}
\hline Parameter & \multicolumn{2}{l}{ Quality of life } & \\
\cline { 2 - 4 } & Estimate & SE & $p$ value \\
\hline Empowerment & 0.057 & 0.009 & $<0.001$ \\
TSSQN & 0.007 & 0.012 & 0.574 \\
TSSQS & -0.009 & 0.011 & 0.410 \\
Stigma & -0.004 & 0.012 & 0.771 \\
Intervention effect at baseline (intervention vs. control) & -0.051 & 0.108 & 0.371 \\
Time (in months) & & & $<0.001$ \\
Intervention $\times$ time & & & $<0.001$ \\
Estimated difference in improvement in QoL from baseline (intervention vs. control) & \\
3 months follow up & 4.310 & 0.610 & $<0.001$ \\
6 months follow up & 4.788 & 0.642 & $<0.001$ \\
\hline
\end{tabular}

$S E$ standard error interventions for stigma reduction [69, 70]. Further, it has been suggested that the limited interventions were available to combat different forms of stigma and discrimination experienced by HIV infected people [70]. Therefore, our study applied an empowerment strategy to overcome and resist the manifestation of discrimination and stigma among HIV infected people with the adaptation of local context and culture. In addition, empowerment would help to defeat symptoms of stigma among HIV infected people and our trial found that increased empowerment could significantly reduce stigma. This result was similar to a systematic review and meta-synthesis which highlighted increased adherence were linked with decreased stigma [71].

The intervention was found to be significantly effective for improving QoL. Systematic reviews based on different interventions and observational studies which focused on
QoL of HIV infected populations showed inconclusive results [72-75]. This might be due to the use of different measurement scales, sampling process and sample size, culture and context of study settings in different studies. Therefore, we used different analytical approaches and pretested cultural and contextual appropriateness of the intervention manual which could improve the reliability of the outcome.

Existing cross-sectional studies from China suggested that social support and stigma were correlated with QoL and social support was the moderator of the impact of stigma on QoL of HIV infected people [76, 77]. A systematic review revealed that social support and ART adherence were associated with QoL of HIV infected people [78]. However, in this study, social support, stigma and ART adherence were not statistically significant but increased stigma showed negative effects on QoL. The 
small sample size could be the possible reason that we could not established statistically significant results with these variables.

This study has several strengths. First, this randomized controlled trial was based on real world study settings that represent the ART receiving HIV infected individuals. Second, the intervention attendance and retention rate was high which signifies the feasibility and acceptability of the intervention for HIV infected people. Third, this is the first multidimensional outcome related trial in Nepal for HIV infected people. Fourth, the intervention process followed extensive quality control and results on all study effects were large. We followed rigorous analysis methods and reported the effect sizes of all the outcomes. Lastly, randomization process, pretested tools, intervention manual and blinding analysis assessor increased the validity and reliability.

This study has several limitations. First, we delivered the intervention in a single study setting and participants were not blinded, which could lead to contamination. However, we detected highly significant differences among the intervention and control group at 3- and 6-months after baseline. Second, the intervention was delivered by skilled personnel with public health graduate degrees, thus limited availability of skilled personnel would limit its sustainability and accessibility. Next, the intervention was led by less trained service providers need to be assessed. Third, we did not assess and determine if the benefit of the intervention was sustainable; we only assessed the outcome at 3- and 6-months follow up. Fourth, outcomes of subgroup analyses were difficult to validate due to the small sample size. Finally, we did not cover the economic and biomarker aspects.

\section{Conclusions}

Rigorously designed intervention indicates that empowerment intervention can increase QoL of HIV infected people. Further, it could be useful to reduce stigma and increase their social support network. Findings could be utilized at regular service settings for its sustainability and long-term effect. Although the intervention effects on secondary outcomes were detected, we recommend evaluating in future multicenter studies with large sample sizes for monitoring the long term effects.

Acknowledgments This study was a part of the thesis of the first author for fulfilling the requirements of a Ph.D degree in Epidemiology at Prince of Songkla University, Thailand which is partially supported by the "Discipline of Excellence in Epidemiology (phase 2): Asia Mentoring Institute in Research". We would like to thank the Graduate School, Prince of Songkla University, Thailand for providing research funds (Grant No. 950/1538). We thank the participants who volunteered to participate in this study. We also thank staff of the ART center for their help, regular support and coordination. The authors are grateful to Mr. Edward McNeil for his efforts with scientific editing.

Authors' Contributions DNB designed the intervention package and conceptualized the study, designed the protocol, coordinated and managed all the aspects of study procedures, reviewed study data, analyzed and interpreted the data, and drafted the manuscript. TL participated in designing the protocol, designed the study, reviewed study data, and reviewed and edited the final manuscript.

Funding Graduate School, Prince of Songkla University, Thailand for providing research funds (Grant No. 950/1538).

\section{Compliance with Ethical Standards}

Conflict of Interest The authors declare that they have no conflict of interest.

Ethical Approval Research Ethics Committee, Faculty of Medicine, Prince of Songkla University, Thailand (57-0146-18-5) and Institutional Ethical Review Committee of Sukraraj Tropical and Infectious Disease Hospital (STIDH), Nepal (063/071/72) approved this study. All procedures performed in studies involving human participants were in accordance with the ethical standards of the institutional research committee and with the 1964 Helsinki declaration and its later amendments or comparable ethical standards.

Informed Consent Informed consent was obtained from all individual participants included in the study.

Open Access This article is distributed under the terms of the Creative Commons Attribution 4.0 International License (http://crea tivecommons.org/licenses/by/4.0/), which permits unrestricted use, distribution, and reproduction in any medium, provided you give appropriate credit to the original author(s) and the source, provide a link to the Creative Commons license, and indicate if changes were made.

\section{References}

1. UNAIDS \& WHO. Joint United Nations Programme on HIV/ AIDS: Global report: UNAIDS report on the global AIDS epidemic 2010. WHO Publications; 2011.

2. UNAIDS. Getting to zero: 2011-2015 strategy. Joint United Nations Programme on HIV/AIDS. UNAIDS Geneva; 2010.

3. UNAIDS. Global report: UNAIDS report on the global AIDS epidemic 2010. UNAIDS Geneva; 2010.

4. NCASC. Nepal Country Progress Report. Kathmandu: National Centre for AIDS and STD Control. Ministry of Health and Population, Government of Nepal; 2015.

5. Naghavi M, Wang H, Lozano R, et al. Global, regional, and national age-sex specific all-cause and cause-specific mortality for 240 causes of death, 1990-2013: a systematic analysis for the Global Burden of Disease Study 2013. Lancet. 2015;385(9963): 117-71.

6. Hosek S, Brothers J, Lemos D, The Adolescent Medicine Trials Network for HIV, AIDS Interventions. What HIV-positive young women want from behavioral interventions: a qualitative approach. AIDS Patient Care STDs. 2012;26(5):291-7.

7. Beyrer C, Baral SD, Walker D, Wirtz AL, Johns B, Sifakis F. The expanding epidemics of HIV type 1 among men who have sex 
with men in low-and middle-income countries: diversity and consistency. Epidemiol Rev. 2010;32:137-51.

8. Baral S, Sifakis F, Cleghorn F, Beyrer C. Elevated risk for HIV infection among men who have sex with men in low-and middleincome countries 2000-2006: a systematic review. PLoS Med. 2007;4(12):e339.

9. Mathers BM, Degenhardt L, Ali H, et al. HIV prevention, treatment, and care services for people who inject drugs: a systematic review of global, regional, and national coverage. Lancet. 2010;375(9719):1014-28.

10. Nakagawa F, Lodwick RK, Smith CJ, et al. Projected life expectancy of people with HIV according to timing of diagnosis. AIDS. 2012;26(3):335-43.

11. Collaboration Antiretroviral Therapy Cohort. Life expectancy of individuals on combination antiretroviral therapy in high-income countries: a collaborative analysis of 14 cohort studies. Lancet. 2008;372(9635):293-9.

12. Steward WT, Herek GM, Ramakrishna J, et al. HIV-related stigma: adapting a theoretical framework for use in India. Soc Sci Med. 2008;67(8):1225-35.

13. Carballo E, Cadarso-Suárez C, Carrera I, et al. Assessing relationships between health-related quality of life and adherence to antiretroviral therapy. Qual Life Res. 2004;13(3):587-99.

14. Clingerman E. Physical activity, social support, and health-related quality of life among persons with HIV disease. J Community Health Nurs. 2004;21(3):179-97.

15. Jin Y, Liu Z, Wang X, et al. A systematic review of cohort studies of the quality of life in HIV/AIDS patients after antiretroviral therapy. Int J STD AIDS. 2014;25(11):771-7.

16. Do AN, Rosenberg ES, Sullivan PS, et al. Excess burden of depression among HIV-infected persons receiving medical care in the United States: data from the medical monitoring project and the behavioral risk factor surveillance system. PLoS One. 2014;9(3):e92842.

17. Miners A, Phillips A, Kreif N, et al. Health-related quality-of-life of people with HIV in the era of combination antiretroviral treatment: a cross-sectional comparison with the general population. Lancet HIV. 2014;1(1):e32-40.

18. Van Dyk AC. Differences between patients who do and do not adhere to antiretroviral therapy. J Assoc Nurs AIDS Care. 2011;22(4):269-82.

19. Amico KR, Harman JJ, Johnson BT. Efficacy of antiretroviral therapy adherence interventions: a research synthesis of trials, 1996 to 2004. J Acquir Immune Defic Syndr. 2006;41(3): 285-97.

20. Laurent C, Gueye NFN, Ndour CT, et al. Long-term benefits of highly active antiretroviral therapy in Senegalese HIV-1-infected adults. J Acquir Immune Defic Syndr. 2005;38(1):14-7.

21. Stringer JS, Zulu I, Levy J, et al. Rapid scale-up of antiretroviral therapy at primary care sites in Zambia: feasibility and early outcomes. JAMA. 2006;296(7):782-93.

22. Mills EJ, Nachega JB, Bangsberg DR, et al. Adherence to HAART: a systematic review of developed and developing nation patient-reported barriers and facilitators. PLoS Med. 2006;3(11):e438.

23. Deblonde J, De Koker P, Hamers FF, Fontaine J, Luchters S, Temmerman M. Barriers to HIV testing in Europe: a systematic review. Eur J Public Health. 2010;20(4):422-32.

24. Wouters E, Van Damme W, van Rensburg D, Masquillier C, Meulemans H. Impact of community-based support services on antiretroviral treatment programme delivery and outcomes in resource-limited countries: a synthetic review. BMC Health Serv Res. 2012;12(1):194.

25. Qiao S, Li X, Stanton B. Social support and HIV-related risk behaviors: a systematic review of the global literature. AIDS Behav. 2014;18(2):419-41.
26. Stein J, Lewin S, Fairall L, et al. Building capacity for antiretroviral delivery in South Africa: a qualitative evaluation of the PALSA PLUS nurse training programme. BMC Health Services Res. 2008;8(1):240.

27. Kober K, Van Damme W. Scaling up access to antiretroviral treatment in southern Africa: who will do the job? Lancet. 2004;364(9428):103-7.

28. Kerrigan D, Wirtz A, Baral S, et al. The global HIV epidemics among sex workers. Washington, DC: World Bank Publications; 2012.

29. Wirtz AL, Pretorius C, Beyrer C, et al. Epidemic impacts of a community empowerment intervention for HIV prevention among female sex workers in generalized and concentrated epidemics. PLoS One. 2014;9(2):e88047.

30. Kerrigan DL, Fonner VA, Stromdahl S, Kennedy CE. Community empowerment among female sex workers is an effective HIV prevention intervention: a systematic review of the peer-reviewed evidence from low-and middle-income countries. AIDS Behav. 2013;17(6):1926-40.

31. Hermann K, Van Damme W, Pariyo GW, et al. Community health workers for ART in sub-Saharan Africa: learning from experience-capitalizing on new opportunities. Hum Resour Health. 2009;7:31.

32. Schwartländer B, Stover J, Hallett T, et al. Towards an improved investment approach for an effective response to HIV/AIDS. Lancet. 2011;377(9782):2031-41.

33. Kerrigan D, Kennedy CE, Morgan-Thomas R, et al. A community empowerment approach to the HIV response among sex workers: effectiveness, challenges, and considerations for implementation and scale-up. Lancet. 2015;385(9963):172-85.

34. USAID: Global Health Programs: Progress Report to Congress FY 2012. Washington, DC: USAID; 2013.

35. UKAID: Towards zero infections: the UK's position paper on HIV in the developing world. Department for International Development; 2011.

36. UNAIDS: The Joint United Nations Programme on HIV/AIDS. 90-90-90 An ambitious treatment target to help end the AIDS epidemic; 2014. JC2684.

37. Valdiserri RO. HIV/AIDS stigma: an impediment to public health. Am J Public Health. 2002;92(3):341.

38. UNICEF: Building systems of protection for children affected by HIV. AIDS in Lesotho: report of an assessment of programming in Lesotho for families and children affected by HIV/AIDS; 1999.

39. Wu L, Li X. Community-based HIV/AIDS interventions to promote psychosocial well-being among people living with HIV/ AIDS: a literature review. Health Psychol Behav Med Open Access J. 2013;1(1):31-46.

40. Beard J, Feeley F, Rosen S. Economic and quality of life outcomes of antiretroviral therapy for HIV/AIDS in developing countries: a systematic literature review. AIDS Care. 2009;21(11):1343-56.

41. Rogers EM. A prospective and retrospective look at the diffusion model. J Health Commun. 2004;9(S1):13-9.

42. Bandura A. Social-learning theory of identificatory processes. Handb social Theory Res. 1969;213:262.

43. Bandura A, McClelland DC. Social learning theory. Englewood Cliffs: Prentice-Hall; 1977.

44. Brothers J, Harper GW, Fernandez MI, Hosek SG, The Adolescent Trials Network for HIV/AIDS Interventions. EVOLUTION-Taking charge and growing stronger: the design, acceptability, and feasibility of a secondary prevention empowerment intervention for young women living with HIV. AIDS Patient Care STDs. 2014;28(1):33-42.

45. Ewart CK. Social action theory for a public health psychology. Am Psychol. 1991;46(9):931. 
46. Fisher JD, Smith L. Secondary prevention of HIV infection: the current state of prevention for positives. Curr Opin HIV AIDS. 2009;4(4):279.

47. Freire P. Pedagogy of the oppressed. New York: Continuum International Publishing Group; 2000.

48. Gutiérrez L, Oh HJ, Gillmore MR. Toward an understanding of (em) power (ment) for HIV/AIDS prevention with adolescent women. Sex Roles. 2000;42(7-8):581-611.

49. Gutierrez LM. Working with women of color: an empowerment perspective. Soc Work. 1990;35(2):149-53.

50. Rotheram-Borus MJ, Swendeman D, Lee S-J, Li L, Amani B, Nartey M. Interventions for families affected by HIV. Trans1 Behav Med. 2011;1(2):313-26.

51. Zimmerman MA. Psychological empowerment: Issues and illustrations. Am J Community Psychol. 1995;23(5):581-99.

52. Zimmerman MA, Rappaport J. Citizen participation, perceived control, and psychological empowerment. Am J Community Psychol. 1988;16(5):725-50.

53. NCASC. Factsheet N4: Antiretroviral Therapy (ART) services in Nepal, 2011. National Centre for AIDS and STD Control. Ministry of Helath and Population, Government of Nepal; 2011.

54. NCASC. Fact sheets on HIV and AIDS, National Center for AIDS and STD Control. Ministry of Helath and Population, Government of Nepal; 2014.

55. NCASC. National Guidelines for Antiretroviral Therapy. National Centre for AIDS and STD Control. Ministry of Helath and Population, Government of Nepal; 2012.

56. Bhatta D, Liabsuetrakul T. Design and feasibility of a social selfvalue intervention package to empower people living with HIV. AIDS Care. 2016. doi:10.1080/09540121.2016.1164293.

57. Bhatta DN, Liabsuetrakul T. Social self-value intervention for empowerment of HIV infected people using antiretroviral treatment: a randomized controlled trial. BMC Infect Dis. 2016;16(1): 1 .

58. Harper GW, Contreras R, Bangi A, Pedraza A. Collaborative process evaluation: enhancing community relevance and cultural appropriateness in HIV prevention. J Prev Interv Community. 2003;26(2):53-69.

59. Larsen DL, Attkisson CC, Hargreaves WA, Nguyen TD. Assessment of client/patient satisfaction: development of a general scale. Eval Progr Plan. 1979;2(3):197-207.

60. WHOQOL HIV Group. WHOQOL-HIV for quality of life assessment among people living with HIV and AIDS: results from the field test. AIDS Care. 2004;16(7):882-9.

61. Sarason IG, Sarason BR, Shearin EN, Pierce GR. A brief measure of social support: practical and theoretical implications. J Soc Pers Relatsh. 1987;4(4):497-510.

62. Genberg BL, Kawichai S, Chingono A, et al. Assessing HIV/ AIDS stigma and discrimination in developing countries. AIDS Behav. 2008;12(5):772-80.

63. Rogers ES, Chamberlin J, Ellison ML, Crean T. A consumerconstructed scale to measure empowerment among users of mental health services. Psychiatr Serv. 1997;48(8):1042-7.

64. Snijders TA. Multilevel analysis. Berlin: Springer; 2011.
65. R Development Core Team. R: a language and environment for statistical computing. Vienna: $\mathrm{R}$ Foundation for Statistical Computing. ISBN 3-900051-07-0. http://www.R-project.org.

66. NHS. Code of confidentiality. http://webarchive.nationalarchives. gov.uk/20130107105354/http://www.dh.gov.uk/prod_consum_dh/ groups/dh_digitalassets/@dh/@en/documents/digitalasset/dh_4069 254.pdf (2014). Accessed 13 June 2014.

67. Nakimuli-Mpungu E, Wamala K, Okello J, et al. Group support psychotherapy for depression treatment in people with HIV/AIDS in northern Uganda: a single-centre randomised controlled trial. Lancet HIV. 2015;2(5):e190-9.

68. McCambridge J, Kypri K, Elbourne D. In randomization we trust? There are overlooked problems in experimenting with people in behavioral intervention trials. $\mathrm{J}$ Clin Epidemiol. 2014;67(3):247-53.

69. Stangl AL, Lloyd JK, Brady LM, Holland CE, Baral S. A systematic review of interventions to reduce HIV-related stigma and discrimination from 2002 to 2013: how far have we come? J Int AIDS Soc. 2013;16(3):18734.

70. Loutfy M, Tharao W, Logie C, et al. Systematic review of stigma reducing interventions for African/Black diasporic women. J Int AIDS Soc. 2015;18(1):19835.

71. Katz IT, Ryu AE, Onuegbu AG, et al. Impact of HIV-related stigma on treatment adherence: systematic review and metasynthesis. J Int AIDS Soc. 2013;16(3):18640.

72. Neto MG, Conceição CS, Carvalho VO, Brites C. Effects of combined aerobic and resistance exercise on exercise capacity, muscle strength and quality of life in HIV-infected patients: a systematic review and meta-analysis. PLoS One. 2015;10(9):e0138066.

73. Bateganya MH, Amanyeiwe U, Roxo U, Dong M. Impact of support groups for people living with hiv on clinical outcomes: a systematic review of the literature. J Acquir Immune Defic Syndr. 2015;68:S368-74.

74. Medley A, Bachanas P, Grillo M, Hasen N, Amanyeiwe U. Integrating prevention interventions for people living with HIV into care and treatment programs: a systematic review of the evidence. J Acquir Immune Defic Syndr. 2015;68:S286-96.

75. Bateganya MH, Dong M, Oguntomilade J, Suraratdecha C. The impact of social services interventions in developing countries: a review of the evidence of impact on clinical outcomes in people living with HIV. J Acquir Immune Defic Syndr. 2015;68:S357-67.

76. Rao D, Chen W, Pearson C, et al. Social support mediates the relationship between HIV stigma and depression/quality of life among people living with HIV in Beijing, China. Int J STD AIDS. 2012;23(7):481-4.

77. Wu X, Chen J, Huang H, Liu Z, Li X, Wang H. Perceived stigma, medical social support and quality of life among people living with HIV/AIDS in Hunan, China. Appl Nurs Res. 2015;28(2):169-74.

78. Degroote S, Vogelaers D, Vandijck DM. What determines healthrelated quality of life among people living with HIV: an updated review of the literature. Arch Public Health. 2014;72(1):40. 